\title{
Age-Friendly Communities Matter for Older People's Well-Being
}

\author{
Anna P. Nieboer ${ }^{1} \cdot$ Jane M. Cramm $^{1}$
}

Published online: 20 October 2017

(C) The Author(s) 2017. This article is an open access publication

\begin{abstract}
This study aims to identify relationships between age-friendly environments (in terms of social and physical neighborhood attributes) and older people's overall wellbeing, as well as the underlying instrumental goals to achieve overall well-being. A sample of 945 community-dwelling older adults living in Rotterdam's districts Lombardijen, Lage Land/Prinsenland, Oude Westen, and Vreewijk was asked to complete a questionnaire in 2013. A total of $588(62 \%)$ responded. The majority (56\%) of respondents was female, $19 \%$ had low educational levels, $35 \%$ were married, and $85 \%$ were born in the Netherlands. Mean age was $77.1 \pm 5.3$ (range 70-93) years. Levels of age-friendliness and older people's ability to realize the instrumental goals to achieve overall well-being varied tremendously among neighborhoods, with older people living in less age-friendly communities reporting lower levels of well-being. These differences in well-being resulted especially from differences in affection, behavioral confirmation, and comfort. Highereducated older persons were more critical regarding the domains civic participation, transportation, and communication and information in their neighborhoods, suggesting a socioeconomic gradient in the perceived lack of neighborhood attributes facilitating aging in place. Currently, physical and social neighborhood attributes enabling aging in place seem to satisfy the needs for affection, behavioral confirmation, and comfort in some, but not all, neighborhoods. Levels of age-friendliness in neighborhoods did not explain differences in opportunities for older people to realize the instrumental goals of status and stimulation.
\end{abstract}

Keywords Age friendly - Community - Subjective well-being · Community dwelling · Older people

Jane M. Cramm

cramm@eshpm.eur.nl

Anna P. Nieboer

nieboer@eshpm.eur.nl

1 Erasmus School of Health Policy and Management, Erasmus University Rotterdam, Burgemeester Oudlaan 50, 3000 DR Rotterdam, The Netherlands 


\section{Introduction}

With populations aging rapidly, service providers and policy makers are increasingly aware of the importance of building and maintaining age-friendly communities (Lui et al. 2009). As people age they become frailer, which increases their needs for neighbourhood characteristics enabling them to age in place (Cramm et al. 2016). We can conclude that dependence on neighbourhoods varies with frailty status. We may speak of community when community-dwelling older adults realize multiple well-being goals together (Völker et al. 2007). A community can thus be seen as a collection of multifunctional relationships conditioned by the benefits of membership, as well as opportunities for and ease of goal realization. Living in an environment where people are trustworthy, help each other when needed (even when it is not convenient), and do not try to profit at others' expense is expected to benefit the well-being of community-dwelling older people. The concept of community as multifunctionality in social relations thus refers to dependencies among neighbors to produce well-being. Neighborhood social relationships may be an important resource on which older people can rely to help them age in place.

If people create communities with the expectation of realizing well-being (Völker et al 2007), then the examination of relationships between neighborhood characteristics as conditions under which this realization is more (or less) likely is an interesting approach to determine the importance of age-friendly communities for older people's well-being. Community-dwelling older adults' ability to achieve well-being can be assessed with more specificity via the application of social production function (SPF) theory. SPF theory, developed by Lindenberg (1996) asserts that people produce their own well-being by trying to optimize achievement of universal goals within the constraints they are facing (Nieboer and Lindenberg 2002). In this case, the ability of community-dwelling older adults to produce their own well-being via the resources they have or do not have at their disposal within their neighborhood. Research indeed shows that physical and social neighborhood characteristics can either be protective or harmful for older people's health (Yen et al. 2009). Given that people will generally strive to achieve physical and social well-being within the available set of resources and constraints, the neighborhood also provides them with more (or fewer) opportunities to do so. The "costs" of producing well-being may be very different in different neighborhoods. Distinguishing a number of universal and (hierarchically ordered) instrumental goals allows us to trace how community-dwelling older adults are able to achieve well-being given the availability (or lack of) resources in the neighborhood.

Physical well-being is achieved through the instrumental goals of stimulation and comfort. Stimulation refers to activation which produces arousal, including mental and sensory stimulation and physical effort (Lindenberg 1996; Nieboer and Lindenberg 2002, p. 2). Promoting social and civic participation among older adults within the neighborhood is a potential way to make sure community-dwelling older adults are still able to achieve physical well-being via stimulating activities creating arousal, mental and sensory stimulation and physical activity. Given that neighborhoods differ in the extend and variety of opportunities for older adults to participate in society (Lin 2017) this may also affect their well-being. Comfort refers to the absence of deleterious stimuli, such as fear, pain, hunger, and thirst (Wippler 1987). Feeling secure and protected in the neighborhood may therefore be important resources for older people's comfort in order to achieve physical well-being given that this is expected to help older people feeling less afraid. But also the availability of community support, local shops and health services attending to older people's potential pain, hunger and thirst (e.g. access to social protection schemes, social security programs 
and/or social assistance programs) are expected to be important resources within the neighborhood for older people to realize physical well-being.

Social well-being is achieved by realizing the three instrumental goals of affection, behavioral confirmation and status. Affection refers to love, friendship and emotional support that can either be given by one's partner or children, but can also come from community members. Affective and instrumental support (trust, reciprocity, and social bonds) provided within the neighborhood environment indeed have been identified as important predictors of community-dwelling older people's well-being (Cramm and Nieboer 2015). Poor neighborhood conditions in terms of affection can pose difficulties in obtaining support, especially for community-dwelling older people since they spend a greater proportion of their lives in their neighborhoods. Such poor neighborhood conditions may lead to difficulties in the achievement of social well-being among communitydwelling older people. Neighborhoods with higher levels of affective and instrumental support, in turn, are expected to be beneficial to the well-being of older adults. Behavioral confirmation is about doing the right thing in the eyes of relevant others. Respect and social approval of older people in the community are considered to be important in this regard (WHO 2007). If community members generally respect older people and include them socially, this will positively affect their ability to achieve social well-being. Research indeed shows that social exclusion is an important domain that shapes vulnerability in old age (Schröder-Butterfill and Marianti 2006). Status refers to a relative social ranking based on a person's profession, lifestyle, or specific talents. Earlier research shows that status is the first instrumental goal most likely to be compromised after retirement (Steverink 2001; Cramm and Nieboer 2015). Providing opportunities for volunteering and helping others in the neighborhood may provide an important resource for older people to remain the ability to achieve some sort of status (van Dijk et al. 2013).

In sum, according to the SPF theory people try to optimize their instrumental goals (affection, behavioral confirmation, status, comfort and stimulation) to achieve overall well-being (social and physical well-being) (Lindenberg 1996; Ormel et al. 1999). Substitution among (instrumental) goals is possible, but only to a limited degree. SPF theory facilitates more pronounced understanding of how community-dwelling older adults achieve well-being and how their abilities to realize well-being may be affected by their neighborhoods as well as individual characteristics and resources (Cramm et al. 2013; Oswald et al. 2011; Wiles et al. 2012).

The creation of age-friendly communities requires a "person-environment fit," i.e., congruence among older people's individual needs and resources (e.g., educational level, ethnicity, marital status), physical environmental conditions (e.g., outdoor spaces and buildings, housing, transportation, community support, local shops and health services) and social environmental conditions (e.g., social participation, civic participation, respect, social approval) (Keating et al. 2013; Menec et al. 2011). Little is known about why some communities are more age-friendly than others, and how such communities are related to older people's well-being (Kendig 2003). We lack description of age-friendly neighborhoods and documentation of the effectiveness of age-friendly communities in promoting older people's well-being (Lui et al. 2009). The aim of this study is thus to identify the relationship between an age-friendly environment (in terms of social and physical neighborhood attributes) and older people's well-being, to increase our understanding of how to create age-friendly communities. 


\section{Methods}

\subsection{Participants}

For this study 945 community-dwelling older adults living in 70 neighborhoods covering Rotterdam's districts Lombardijen, Lage Land/Prinsenland, Oude Westen, and Vreewijk were selected to participate in the T1 study (2013). These respondents already filled in a T0 questionnaire in 2011. Stratified sampling took place where people were sampled proportionate to their age (age groups: $70-74,75-79,80-84, \geq 85$ years) and the neighborhoods/districts leading to a total of 430 eligible older people per district. Before the T1 study all addresses were carefully selected for potential drop out caused by death, institutionalization, or moving out of the district. This resulted in a final sample of $n=838$ to be approached again in 2013. We first invited the sample to participate via mail or email. In order to increase the response rate people were offered a 1/5 ticket in the monthly Dutch State Lottery. Those who still did not respond received a reminder by mail or email. A total of $n=371$ responded via mail and 77 via email. Non-responders were then asked to participate via telephone, which led to another 77 older people who filled in the questionnaire by telephone. A final attempt was made through a home visit leading to an additional 86 older people filling in the questionnaire. After a careful quality check three questionnaires were not filled in by the selected respondent. Which led to a final response of $\mathrm{n}=588$.

\subsection{Sample Characteristics}

Table 1 displays the characteristics of the study participants. Of the 558 respondents, $56 \%$ were female, $19 \%$ had low educational levels, $35 \%$ were married, and $85 \%$ were born in the Netherlands. Mean age was $77.1 \pm 5.3$ (range 70-93) years. The mean overall wellbeing score was $2.6 \pm 0.6$ (range $1-4)$.

This study was approved by the ethics committee of Erasmus University Medical Centre of Rotterdam (MEC-2011-197). More information regarding the study design can be found in the study protocol (Cramm et al. 2011).

\subsection{Measures}

Well-being as well as instrumental goals to achieve well-being was assessed with the 15-item version of the Social Production Function Instrument for the Level of Well-Being ("Appendix 1"). This scale has been validated thoroughly and measures overall well-

Table 1 Descriptive statistics for the study population $(n=588)$

$S D$ standard deviation, $S P F-I L$ social production function instrument for the level of wellbeing

\begin{tabular}{lll}
\hline Demographic characteristic & Range & $\%$ or mean (SD) \\
\hline Gender (female) & & $56 \%$ \\
Age (years) & $70-93$ & $77.1(5.3)$ \\
Marital status (married) & & $35 \%$ \\
Ethnic background (Dutch) & & $85 \%$ \\
Education (low) & & $19 \%$ \\
Aging in place & $1-5$ & $1.7(0.7)$ \\
Overall well-being (SPF-IL) & $1-4$ & $2.6(0.6)$ \\
\hline
\end{tabular}


being, as well as levels of physical (comfort, stimulation) and social (behavioral confirmation, affection, and status) well-being (Nieboer et al. 2005). Examples of questions are: "Do you feel that people really love you?" (affection), "Do you feel useful to others?" (behavioral confirmation), "Are you known for the things you have accomplished?" (status), "In the past few months have you felt physically comfortable?" (comfort), and "Do you really enjoy your activities?" (stimulation). Respondents could rate their level of agreement on a four-point scale ranging from never (1) to always (4). The higher the score the greater their well-being. Cronbach's alpha value for overall well-being was .88, indicating good reliability. Cronbach's alpha value for the affection, behavioral confirmation, status, comfort, and stimulation subscales were .86, .66, .77, .89, and .79, respectively. This instrument has been used extensively to investigate older people's wellbeing (e.g. van Dijk et al. 2016; Cramm et al. 2013; Slotman et al. 2015; Cramm and Nieboer 2015).

Aging in place was assessed using statements developed in a previous study utilizing the 2007 World Health Organization (WHO) framework for age-friendly cities and additional aging in place literature (e.g., Lui et al. 2009; van Dijk et al. 2014; "Appendix 2"). Statement comprehensiveness and unambiguity were tested during interviews with older people. After the careful exclusion or rephrasing of overlapping items, 24 items representing the eight domains identified by the WHO for the promotion of active aging (social participation, transportation, outdoor spaces and buildings, housing, civic participation, communication and information, respect and social approval, and community support and health services) were used in the present study. Participants were asked to indicate whether they missed components in their neighborhoods for aging in place on a five-point scale ranging from 1 (not missed at all) to 5 (extremely missed). Mean subscale scores were calculated when responses to at least $50 \%$ of the items were available, and the total scale score was calculated using average subsection scores (when responses to all eight subsections were available). Intercorrelations between subscales ranged between .380 and .627. The composite reliability score of the ageing in place instrument was .91, indicating good reliability.

The questionnaire further investigated participants' age, gender, ethnic background, marital status, and education. Educational level was assessed using a seven-point scale ranging from 1 (primary school or less) to 7 (university degree). We then dichotomized educational level as poor (1; primary school or less) or good $(0$; more than primary school).

\subsection{Statistical Analyses}

Descriptive statistics were used to characterize our study population. The $10 \%$ worst neighborhoods were selected based on aging in place scale scores. The seven neighborhoods with the highest scores on this scale (indicating missing neighborhood attributes) were selected as the "worst" neighborhoods. A total of 103 elderly persons who completed the questionnaire (20\% of all respondents) lived in these neighborhoods. We compared physical, social, and overall well-being scores between these poor neighborhoods and the other $90 \%$ of neighborhoods. Third, we employed bivariate (Pearson and Spearman as appropriate) and multilevel analyses [two-level random-effects model using variance component (identity): level $1=$ individual respondents $(n=588)$, level $2=$ neighborhoods $(n=70)$ ] to investigate relationships between background characteristics, social and physical neighborhood attributes for aging in place that older people missed, and wellbeing (overall and in terms of instrumental goals). Statistical analyses were conducted 
using SPSS software (version 21; IBM Corporation, Armonk, NY, USA). Results were considered to be significant when two-sided $p$ values were $\leq .05$.

\section{Results}

Table 2 shows the well-being of respondents living in the $10 \%$ worst neighborhoods for aging in place and those living in the other neighborhoods. Well-being of older people living in the $10 \%$ worst neighborhoods was significantly lower than that of those living in other neighborhoods ( 2.5 vs. $2.6 ; p \leq .05$ ). This difference was due especially to differences in social well-being (affection and behavioral confirmation), but also to differences in older people's level of comfort (physical well-being). The difference in effect size (Cohen's $d$ ) fell between small (0.2) and medium (0.5) (Cohen 1988).

Bivariate analyses showed that overall well-being was associated positively with female gender $(p=.005)$ and Dutch ethnic background $(p=.034)$, and negatively with low educational level $(p=.010)$ and missing neighborhood attributes $(p<.001$; Table 3$)$. Significant negative relationships were also found between missing neighborhood attributes and the instrumental goals of comfort $(r=-.17, p<.001)$, affection $(r=-.26$, $p<.001)$, and behavioral confirmation $(r=-.17, p<.001)$.

The results of multilevel analyses are displayed in Table 4. Gender $(p \leq .01)$, educational level $(p \leq .01)$, and aging in place scores $(p \leq .01)$ predicted older people's overall well-being. Aging in place scores predicted the ability to achieve comfort $(p \leq .001)$, affection ( $p \leq .001)$, and behavioral confirmation $(p \leq .01)$, but not stimulation or status. Stimulation was determined primarily by educational level $(p \leq .01)$ and ethnic background $(p \leq .05)$, and status was determined mainly by educational level ( $p \leq 005)$.

In addition, we investigated the relationships between (instrumental goals to realize) well-being and the eight domains identified by the WHO to promote active aging (results shown in Table 5). In general, most ageing in place domains were related to the instrumental goals of comfort, affection, and behavioral confirmation. Status was related significantly to transportation, civic participation, and communication and information, and stimulation was related only to civic participation. In addition to the small number of WHO domains found to be related to status and stimulation, the direction of these relationships

Table 2 Instrumental goals to achieve well-being according to neighborhood ranking for aging in place

\begin{tabular}{|c|c|c|c|c|}
\hline & $\begin{array}{l}10 \% \text { worst neighborhoods } \\
\text { for aging in place } n=103\end{array}$ & $\begin{array}{l}90 \% \text { best neighborhoods } \\
\text { for aging in place } n=423\end{array}$ & Cohen's $d$ & $p$ value \\
\hline \multicolumn{5}{|l|}{ Social well-being } \\
\hline Affection & $2.48(0.55)$ & $2.72(0.70)$ & -0.381 & .001 \\
\hline $\begin{array}{l}\text { Behavioral } \\
\text { confirmation }\end{array}$ & $2.68(0.75)$ & $2.85(0.69)$ & -0.235 & .034 \\
\hline Status & $1.96(0.68)$ & $1.90(0.73)$ & 0.085 & .444 \\
\hline \multicolumn{5}{|l|}{ Physical well-being } \\
\hline Comfort & $2.49(0.84)$ & $2.74(0.82)$ & -0.301 & .006 \\
\hline Stimulation & $2.82(0.79)$ & $2.72(0.70)$ & 0.134 & .235 \\
\hline Overall well-being & $2.48(0.55)$ & $2.59(0.53)$ & -0.203 & .047 \\
\hline
\end{tabular}

Data are expressed as mean (standard deviation). Results are based on $t$ tests 


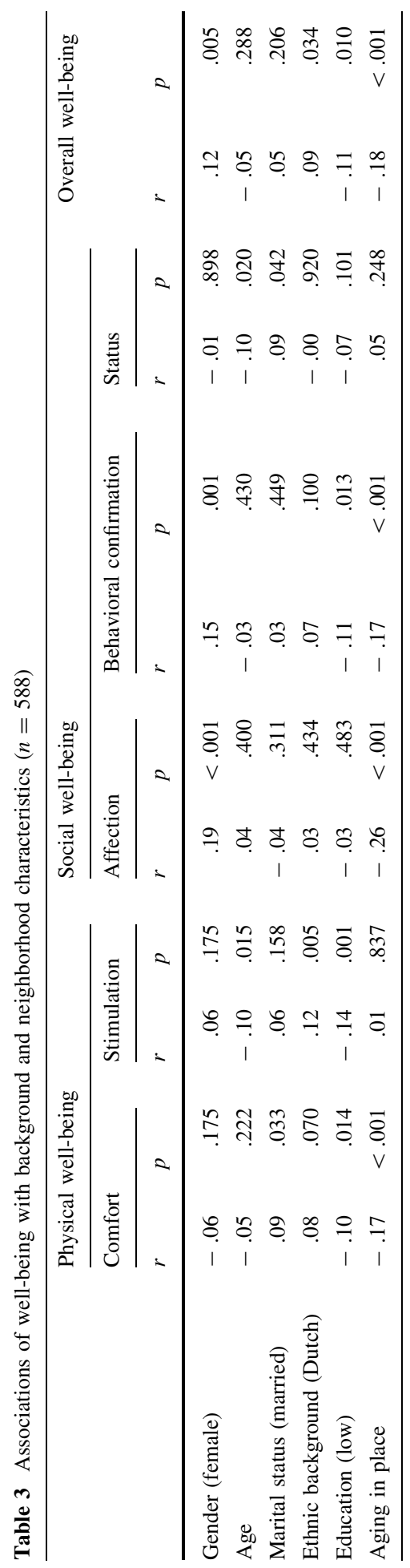




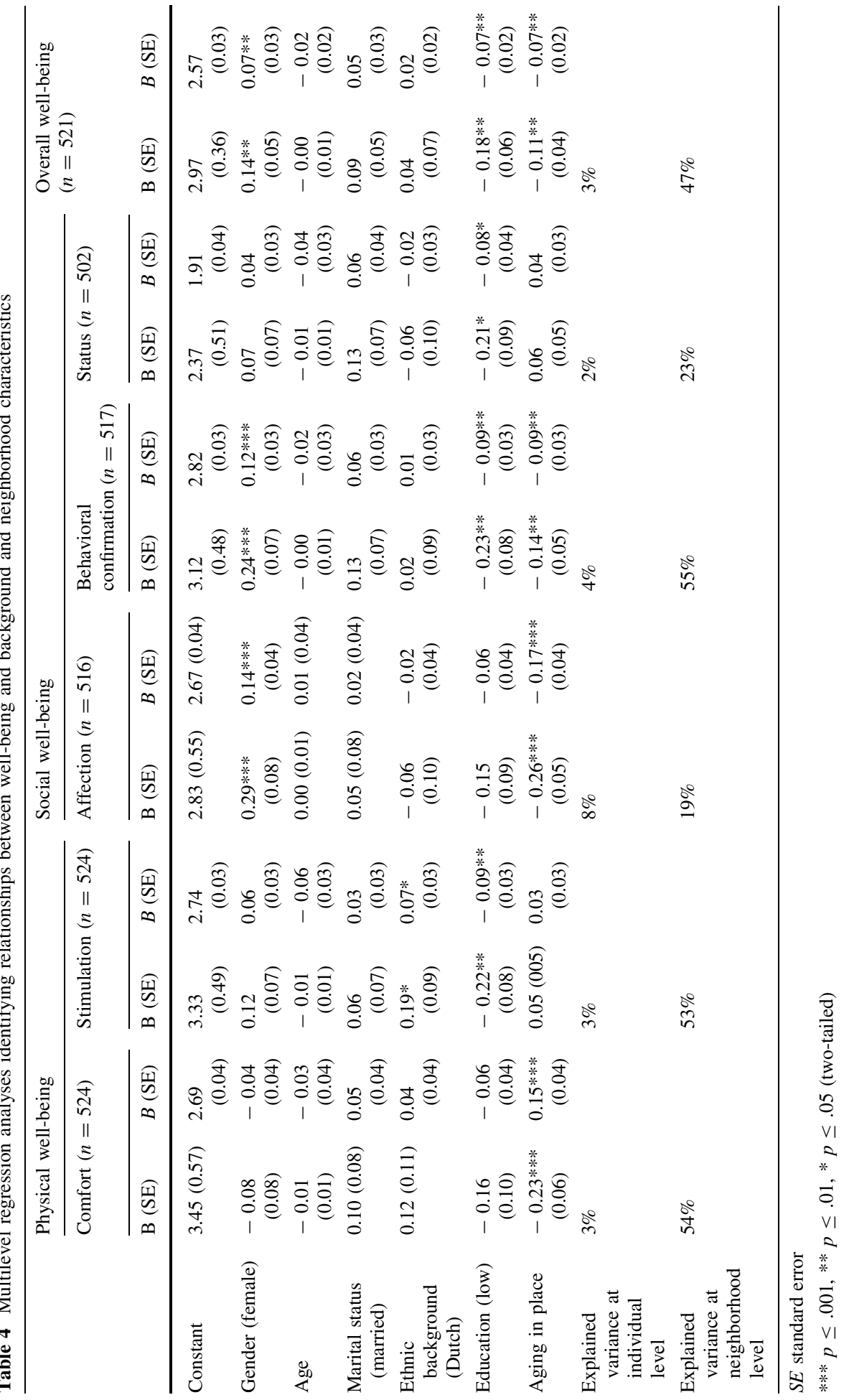


Table 5 Associations of well-being with the eight ageing in place domains $(n=588)$

\begin{tabular}{|c|c|c|c|c|c|}
\hline & \multicolumn{2}{|c|}{ Physical well-being } & \multicolumn{3}{|c|}{ Social well-being } \\
\hline & Comfort & Stimulation & Affection & $\begin{array}{l}\text { Behavioral } \\
\text { confirmation }\end{array}$ & Status \\
\hline Outdoor spaces and buildings & $-0.15 * * *$ & 0.01 & $-0.16^{* * *}$ & $-0.15^{* * *}$ & 0.06 \\
\hline Transportation & $-0.09 *$ & 0.03 & $-0.14 * * *$ & $-0.12 * *$ & $0.10^{*}$ \\
\hline Housing & $-0.09 *$ & 0.07 & $-0.11^{* *}$ & -0.05 & 0.04 \\
\hline Social participation & $-0.16^{* * *}$ & -0.01 & $-0.18^{* * *}$ & $-0.13^{* *}$ & 0.00 \\
\hline Respect and social approval & $-0.14 * * *$ & 0.03 & $-0.32 * * *$ & $-0.21 * * *$ & 0.02 \\
\hline Civic participation & -0.06 & $0.09^{*}$ & $-0.14 * * *$ & -0.02 & $0.14^{* *}$ \\
\hline Communication and information & $-0.10 *$ & 0.05 & $-0.10 *$ & -0.06 & $0.09 *$ \\
\hline $\begin{array}{l}\text { Community support and health } \\
\text { services }\end{array}$ & $-0.23 * * *$ & -0.05 & $-0.20 * * *$ & $-0.18^{* * *}$ & 0.01 \\
\hline
\end{tabular}

$* * * p \leq .001, * * p \leq .01, * p \leq .05$ (two-tailed)

differed from the results of the multilevel analyses. Whereas missing neighborhood attributes were related negatively to older people's well-being overall, the relationships of these sub-dimensions to status and stimulation were positive. Those reporting higher levels of status and stimulation were more critical of their neighborhoods in the domains of civic participation, transportation, and communication and information. However, only highly educated older persons were more critical of the age-friendliness of communities in these three domains, suggesting a socioeconomic gradient in perceptions about missing neighborhood attributes for aging in place.

\section{Discussion}

In recent years, growing political and policy interest has targeted community environments and their potential to support aging in place. The increasing importance of environmental gerontology (Kendig 2003; Phillips et al. 2005; Wahl et al. 2003) has further intensified discussions related to the development of such communities. However, theory about why some communities are more age-friendly than others and how such communities are related to older people's well-being is lacking (Lui et al. 2009). This study thus aimed to identify relationships between age-friendly environments and older people's well-being, to increase understanding of how people experience their neighborhoods' facilitation of aging in place and of how to create age-friendly communities. This research provided empirical quantitative evidence that aging in place (in terms of physical and social neighborhood environments) indeed matters for older people's well-being, even after accounting for socioeconomic and background characteristics.

Our findings indicate that age-friendly neighborhoods in the districts of Rotterdam are likely to satisfy older people's need for affection, behavioral confirmation, and comfort. In age-friendly communities, affection is satisfied when older people feel liked, trusted, accepted, and understood; they feel that others empathize with them, are willing to help without expecting anything in return, and display emotional and/or physical closeness (e.g., hugging). Affection also involves the feeling that one's well-being is intertwined with that of one's neighbors, and is not contingent on one's assets (status) or actions (behavioral 
confirmation). Behavioral confirmation is satisfied by doing and being good, contributing usefully to a common goal and being part of the community; it is characterized by the perception that one is doing the "right" thing, not only on one's own eyes, but also in those of one's neighbors. This goal is thus achieved by older people's actions in their neighborhoods, rather than the types of person that they are (affection) or their resources and assets (status) (van Bruggen 2001). Because community involves dependencies among neighbors to produce well-being, (multifunctional) social relationships may be an important resource for older people's ability to age in place. The satisfaction of affection and behavioral confirmation is thus particularly relevant in improving community dwelling older persons' well-being.

The ability to stimulate comfort among community-dwelling older people also differs among neighborhoods. This applies to both social as well as physical neighbourhood attributes and especially community support and health services. Earlier research also found that neighbourhood attributes affect physical health (Jonker et al. 2014; Kawachi et al. 2008; van Lenthe et al. 2005). Relationships, for example, were found between the physical ageing in place domain outdoor spacing, the social neighbourhood attribute safety and individual health (e.g. physical activity, physical health, and life expectancy) (Beenackers et al. 2013; Jonker et al. 2014).

Physical and social neighborhood attributes facilitating aging in place seem to provide limited opportunities for older people to realize the instrumental goals of status and stimulation. Although all eight domains have been shown to be important for aging in place (Lui et al. 2009; Menec et al. 2011), they may not all be equally important for older people's well-being. Although we found that all eight domains mattered for older people's overall well-being, as well as the instrumental goals of affection, behavioral confirmation, and comfort, civic participation was the only domain related to stimulation and civic participation, transport, and communication and information predicted status. Status is known to be linked to lower-order means to achieve well-being, such as wealth, education, and work (Nieboer et al. 2005). This association implies that older people with higher educational and income levels who continue to do community and/or voluntary work are those reporting the highest status levels. These people may expect more from their neighborhoods in terms of the ability to achieve well-being, such as transportation, civic participation, communication, and education. Those with lower educational and income levels who do not participate in community activities may expect less from neighborhoods in terms of these specific attributes. The same relationship is expected to apply to stimulation and civic participation; those reporting higher levels of stimulation were most critical about civic participation in the neighborhood.

This study also clearly showed that levels of age-friendliness and community-dwelling older people's abilities to realize instrumental goals to achieve overall well-being varied tremendously among neighborhoods, with older people living in less age-friendly communities reporting lower levels of well-being. These results support our previous finding that older people living in the more socially deprived neighborhoods (in terms of social belonging and social cohesion) reported lower levels of well-being (Cramm and Nieboer 2015). The current study adds to this knowledge by investigating the relationship between an age-friendly environment (in terms of physical and social neighborhood attributes) and the well-being of community-dwelling older people. Older people living in less agefriendly communities have particular difficulty in optimizing the achievement of the instrumental goals of behavioral confirmation, comfort, and affection, as well as overall well-being. 
In addition to the importance of improving (or protecting loss of) well-being among community-dwelling older people, preventing or at least delaying institutionalization is needed. Steverink (2001) showed that deterioration of older people's ability to optimize the instrumental goals of comfort and affection is the most likely predictor of living independently. This calls for measures that go beyond physical instrumental goals only (e.g., comfort). To effectively avoid institutionalization policy makers should also support older people's social instrumental goals (e.g., affection) (Cramm and Nieboer 2015), for example via the creation of age-friendly communities. Given the results of the present study that living in an age-friendly community supports optimization of instrumental goals to achieve overall well-being, behavioral confirmation, comfort, and affection, and based on the work of Steverink (2001), people living in less age-friendly communities are expected to be at particular risk of institutionalization. This risk further supports the need for investment in age-friendly communities.

By distinguishing different goals to realize well-being, the aim of this study was to trace the consequences of living in more or less age-friendly environments for the well-being of community-dwelling older people, and thereby to identify the changes needed to protect further deterioration of their well-being (Cramm and Nieboer 2015). To improve wellbeing in a time of aging populations, policy makers and governments should create agefriendly communities by investing in physical and social neighborhood attributes, and especially by identifying opportunities to enable the realization of status and stimulation in neighborhoods. The achievement of status is probably more difficult, as it is known to be the first instrumental goal to decrease over time as people age (Cramm and Nieboer 2015; Steverink 2001). Status is known to be affected by resources such as education, socioeconomic status, and the possession of rare skills (activities that make a person stand out, especially those related to work) (Nieboer 1997). A person's status drops immediately after retirement (Steverink 2001). Regression analyses have shown that education remains the main source of status among community-dwelling older people. Education has been identified as an asset that equips people with resources (knowledge, but also social networks formed during education and subsequent employment) and social skills, such as being an influential person and being known for the things one has accomplished, which are the main status indicators. In the present study, stimulation was affected positively by a higher educational level and by being born in the Netherlands. People with lower educational levels and those not born in the Netherlands may experience more difficulty engaging in activities that are both challenging and enjoyable. Researchers applying SPF theory have identified the importance of resources such as physical and mental capacities for the creation of stimulation (Nieboer 1997). People with lower educational levels and immigrants are known to report lower levels of physical and mental health (Reijneveld 1998; Schellingerhout 2004), which may explain their difficulties in the realization of stimulation.

Our research has several limitations. Because of the cross-sectional design we were not able to draw causal conclusions. The relationship between aging in place and well-being must be recognized as dynamic; people whose well-being deteriorates with age are expected to be more dependent on their neighborhoods, whereas those in good health and/ or well-being are expected to be less dependent (van Dijk et al. 2014). A longitudinal study design is needed to examine the relationship between aging and place and well-being over time. Furthermore, this study investigated only a population of older residents in Rotterdam. In a time when aging in place tops the priority lists of health research and policy, an understanding of differences among municipalities and their effects on older people's well- 
being is also needed. Thus, future nationwide research and/or comparisons among countries is warranted.

Whereas most studies of age-friendly communities to date have been essentially descriptive or narrative and based primarily on qualitative analyses (Lui et al. 2009; Menec et al. 2011), this study empirically shows the importance of age-friendly communities for the well-being of community-dwelling older people. Our findings have implications for policy makers and service providers aiming to build and maintain age-friendly communities. To protect deterioration of community-dwelling older adult's well-being, investment in social and physical neighborhood attributes is important. A focus on how community environments can support aging in place and well-being is timely, given that populations are aging rapidly, and policy makers and service providers struggle with the effective and efficient creation of age-friendly communities. These findings could have important implications that contribute to the reduction of a major community burden. A longitudinal follow-up study examining a variety of settings is required to investigate possible causal pathways, and to identify differences among municipalities and their effects on older people's well-being over time.

Acknowledgements Funding was provided by The Netherlands Organization for Health Research and Development (ZonMw) (Project Number 314030201).

Open Access This article is distributed under the terms of the Creative Commons Attribution 4.0 International License (http://creativecommons.org/licenses/by/4.0/), which permits unrestricted use, distribution, and reproduction in any medium, provided you give appropriate credit to the original author(s) and the source, provide a link to the Creative Commons license, and indicate if changes were made.

\section{Appendix 1: The 15-Item Version of the Social Production Function Instrument for the Level of Well-Being (SPF-IL)}

I will ask you a number of questions about how you feel. These questions refer to the past 3 months. For your answer, will you please choose between NEVER, SOMETIMES, OFTEN or ALWAYS? If you HARDLY EVER have that feeling you can answer NEVER. If you ALMOST ALWAYS feel that way, answer ALWAYS. Use whichever answer is CLOSEST to the way you feel, NEVER, SOMETIMES, OFTEN or ALWAYS.

\section{Affection}

1. Do people pay attention to you?

2. Do people help you if you have a problem?

3. Do you feel that people really love you?

\section{Behavioral Confirmation}

4. There are situations in which we deal with groups of people, for example at home, at work or during our leisure time. Do others appreciate your role in the group?

5. Do people find you reliable?

6. Do you feel useful to others? 


\section{Status}

7. Do people think you do better than others?

8. Do people find you an influential person?

9. Are you known for the things you have accomplished?

\section{Comfort}

In the past few months have you felt:

10. ...relaxed?

11. ...in good health?

12. ...physically comfortable?

\section{Stimulation}

13. Are your activities challenging to you?

14. Do you really enjoy your activities?

15. How often are you fully concentrated when doing something?

\section{Appendix 2: Instrument to Asses Missing Neighborhood Attributes to Age in Place}

Respondents were asked what they miss in their neighborhood to age in place on a five point scale ranging from 1 (not missed at all) to 5 (extremely missed).

\section{Outdoor Spaces and Buildings}

A clean and green neighbourhood.

A neighbourhood with wide sidewalks and safe crosswalks.

Public buildings with elevators that are easily accessible for wheelchairs and walkers.

A safe neighbourhood.

\section{Transportation}

Good public transport.

Sufficient parking spots.

\section{Housing}

Affordable housing.

Suitable housing for older people. 


\section{Social Participation}

A neighbourhood where many social activities are organised.

Affordable activities for older people.

\section{Respect and Social Approval}

A neighbourhood where people have respect for older people.

A neighbourhood where people are willing to help each other whenever necessary.

A neighbourhood with people having the same ethnical background as me (not so much immigrants).

A neighbourhood where people dare to speak up to each other.

A neighbourhood where people great and talk to each other.

\section{Civic Participation}

A neighbourhood with possibilities for voluntary work.

A neighbourhood where older people are involved, for example concerning changes in the neighbourhood.

\section{Communication and Information}

Local newspaper with information about what's going on in the neighbourhood.

Access to internet and internet courses in the neighbourhood.

\section{Community Support and Health Services}

A neighbourhood where home care is easily accessible.

A neighbourhood with the GP and pharmacy at walking distance.

A neighbourhood with places where older people can go for advice and support.

A neighbourhood with volunteers who provide help when necessary.

A neighbourhood with shops and other facilities within walking distance.

\section{References}

Beenackers, M. A., Kamphuis, C. B., Mackenbach, J. P., Burdorf, A., \& van Lenthe, F. J. (2013). Why some walk and others don't: Exploring interactions of perceived safety and social neighborhood factors with psychosocial cognitions. Health Education Research, 28(2), 220-233.

Cohen, J. (1988). Statistical power analysis for the behavioral sciences (2nd ed.). Hillsdale, NJ: Lawrence Earlbaum Associates.

Cramm, J. M., \& Nieboer, A. P. (2015). Social cohesion and belonging predict the well-being of community-dwelling older people. BMC Geriatrics, 15, 30. doi:10.1186/s12877-015-0027-y.

Cramm, J. M., van Dijk, H., Lötters, F., van Exel, J., \& Nieboer, A. P. (2011). Evaluating an integrated neighbourhood approach to improve well-being of frail elderly in a Dutch community: A study protocol. BMC Research Notes, 4, 532. doi:10.1186/1756-0500-4-532. 
Cramm, J. M., van Dijk, H. M., \& Nieboer, A. P. (2013). The importance of neighborhood social cohesion and social capital for the well being of older adults in the community. The Gerontologist, 53(Suppl. 1), $142-152$.

Cramm, J. M., van Dijk, H. M., \& Nieboer, A. P. (2016). The creation of age-friendly environments is especially important to frail older people. Ageing \& Society. doi:10.1017/S0144686X16001240.

Jonker, M. F., van Lenthe, F. J., Donkers, B., Mackenbach, J. P., \& Burdorf, A. (2014). The effect of urban green on small-area (healthy) life expectancy. Journal of Epidemiology and Community Health, 68(10), 999-1002. doi:10.1136/jech-2014-203847.

Kawachi, I., Subramanian, S. V., \& Kim, D. (Eds.). (2008). Social capital and health. Berlin: Springer.

Keating, N., Eales, J., \& Phillips, J. E. (2013). Age-friendly rural communities: Conceptualizing 'best fit'. Canadian Journal on Aging, 32(4), 319-332.

Kendig, H. (2003). Directions in environmental gerontology: A multidisciplinary field. The Gerontologist, $43,611-614$.

Lin, W. (2017). A study on the factors influencing the community participation of older adults in China: based on the CHARLS2011 data set. Health and Social Care in the Community, 25(3), 1160-1168.

Lindenberg, S. (1996). Continuities in the theory of social production functions. In H. Ganzeboom \& S. Lindenberg (Eds.), Verklarende sociologie; opstellen voor Reinhart Wippler. Amsterdam: Thesis Publications.

Lui, C. W., Everingham, J. A., Warburton, J., Cuthill, M., \& Bartlett, H. (2009). What makes a community age-friendly: A review of international literature. Australasian Journal on Ageing, 28(3), 116-121.

Menec, V. H., Means, R., Keating, N., Parkhurst, G., \& Eales, J. (2011). Conceptualizing age-friendly communities. Canadian Journal on Aging, 30(3), 479-493.

Nieboer, A. P. (1997). Life-events and well-being: A prospective study on changes in well-being of elderly people due to a serious illness event or death of the spouse. Amsterdam: Thela Thesis.

Nieboer, A., \& Lindenberg, S. (2002). Substitution, buffers and subjective well-being: A hierarchical approach. In E. Gullone \& R. A. Cummins (Eds.), The universality of subjective well-being indicators (pp. 175-189). Dordrecht, NL: Kluwer Academic Publishers.

Nieboer, A., Lindenberg, S., Boomsma, A., \& van Bruggen, A. C. (2005). Dimensions of well-being and their measurement: The SPF-Il Scale. Social Indicators Research, 73(3), 313-353.

Ormel, J., Lindenberg, S., Steverink, N., \& Verbrugge, L. M. (1999). Subjective well-being and social production functions. Social Indicators Research, 46, 61-90.

Oswald, F., Jopp, D., Rott, C., \& Wahl, H.-W. (2011). Is aging in place a resource for or risk to life satisfaction? The Gerontologist, 51(Suppl. 2), 238-250.

Phillips, D. R., Siu, O.-L., Yeh, A. G. O., \& Cheng, K. H. G. (2005). Ageing and the urban environment. In J. A. Gavin \& D. R. Phillips (Eds.), Ageing and place: Perspectives, policy, practice (pp. 147-163). London: Routledge.

Reijneveld, S. A. (1998). Reported health, lifestyles, and use of health care of first generation immigrants in the Netherlands: Do socioeconomic factors explain their adverse position? Journal of Epidemiology and Community Health, 52, 298-304.

Schellingerhout, R. (2004). Gezondheid en welzijn van allochtone ouderen. Den Haag: Sociaal en Cultureel Planbureau.

Schröder-Butterfill, E., \& Marianti, R. (2006). A framework for understanding old-age vulnerabilities. Ageing \& Society, 26(1), 9-35.

Slotman, A., Cramm, J. M., \& Nieboer, A. P. (2015). The ageing perceptions questionnaire (APQ): An examination of its psychometric properties and development of the shortened APQ among Dutch community-dwelling elders. Health and Quality of Life Outcomes, 13, 54.

Steverink, N. (2001). When and why frail elderly people give up independent living: The Netherlands as an example. Ageing \& Society, 21, 45-69.

van Bruggen, A. C. (2001). Individual production of social well-being. An explanatory study (Dissertation). Interuniversity Center for Social Science Theory and Methodology, University of Groningen, Groningen, The Netherlands.

van Dijk, H. M., Cramm, J. M., Birnie, E., Nieboer, A. P. (2016). Effects of an integrated neighbourhood approach on older people's (health-related) quality of life and well-being. BMC Research Notes, 9, 450.

van Dijk, H. M., Cramm, J. M., \& Nieboer, A. P. (2013). The experiences of neighbour, volunteer and professional support-givers in supporting community dwelling older people. Health and Social Care in the Community, 21(2), 150-158.

van Dijk, H., Cramm, J. M., van Exel, J., \& Nieboer, A. P. (2014). The ideal neighbourhood for ageing in place as perceived by frail and non-frail community-dwelling older people. Ageing \& Society. doi:10. 1017/S0144686X14000622. 
van Lenthe, F. J., Brug, J., \& Mackenbach, J. P. (2005). Neighbourhood inequalities in physical inactivity: The role of neighbourhood attractiveness, proximity to local facilities and safety in the Netherlands. Social Science and Medicine, 60(4), 763-775.

Völker, B., Flap, H., \& Lindenberg, S. (2007). When are neighbourhoods communities? Community in Dutch neighbourhoods. European Sociological Review, 23(1), 99-114.

Wahl, H.-W., Scheidt, R., \& Windley, P. G. (Eds.). (2003). Annual review of gerontology and geriatrics. Vol. 23 ageing in context: Socio-physical environments. New York: Springer.

WHO. (2007). Global age-friendly cities: A guide. ISBN 9789241547307.

Wiles, J. L., Leibing, A., Guberman, N., Reeve, J., \& Allen, R. E. S. (2012). The meaning of 'aging in place' to older people. The Gerontologist, 52(3), 357-366.

Wippler, R. (1987). Kulturelle Ressourcen, Gesellschaflicher Erfolg und Lebensqualitat. In G. Giesen \& H. Haferkamp (Eds.), Soziologie der sozialen Ungleichheit (pp. 221-254). Opladen, Germany: Westdeutscher Verlag.

Yen, I. H., Michael, Y. L., \& Perdue, L. (2009). Neighborhood environment in studies of health of older adults. A systematic review. American Journal of Preventive Medicine, 37(5), 455-463. doi:10.1016/j. amepre.2009.06.022. 